\title{
A Novel Space-Time-Speed Method for Increasing the Passing Capacity with Safety Guaranteed of Railway Station
}

\author{
Junfeng Wang, ${ }^{1}$ Yang Yu, ${ }^{1}$ Renwei Kang, ${ }^{2}$ and Jungang Wang ${ }^{3}$ \\ ${ }^{1}$ State Key Laboratory of Rail Traffic Control and Safety, Beijing Jiaotong University, Shangyuancun, Xizhimenwai, \\ Beijing 100044, China \\ ${ }^{2}$ Signalling and Communications Research Institute, China Academy of Railway Sciences, Beijing 100081, China \\ ${ }^{3}$ Transportation Department of Beijing Railways Bureau, Beijing 100860, China \\ Correspondence should be addressed to Junfeng Wang; w2881@163.com
}

Received 8 December 2016; Accepted 7 February 2017; Published 27 February 2017

Academic Editor: Zhi-Chun Li

Copyright (C) 2017 Junfeng Wang et al. This is an open access article distributed under the Creative Commons Attribution License, which permits unrestricted use, distribution, and reproduction in any medium, provided the original work is properly cited.

A method for improving the passing capacity of a station without adding any track and equipment is proposed in this paper. In the process of handling train routes, by transforming the existing fixed train-approaching locking section into a variable mode, the route locking time is shortened and in-station resource consumption is reduced. This approach improves the capacity of the station. At the same time, delay of the train can be quickly returned to normal. A method of variable train-approaching locking section is discussed; a mathematical model for increasing station passing capacity is shown. Comparison between the impact of a variable train-approaching locking section and a fixed mode on the station passing capacity is shown.

\section{Introduction}

Train routes are controlled by station dispatchers using an interlocking system. A train route is first established; when the train gets close to the locking section, the interlocking system will close the route (called approach locking), in order to guarantee the safety of train operation. After locking, all of the switches, station tracks, and signal machines on the route are in the closed state; the route can not be used by other trains and can not be unblocked within a predetermined delay time. The route is unblocked when the train gets in. The length of the train-approaching locking equates the quantity of block section included in the route [1]. The longer the trainapproaching locking section, the larger the impact on the capacity of the station. The distance of the train-approaching locking section depends on the speed of the train, the maximum service braking distance, train type, the level of control, other line parameters (such as slope), and so forth [2]. Currently, the mode of train-approaching locking specified in the Centralized Traffic Control (CTC) is fixed. The train route is arranged uniformly when train approaches 15th block sections early on and is locked when train approaches 8th block sections regardless of discrepancy of speed and braking ability. The length of 7 front block sections is distance of train running during process of handling train routes, while the length of 8 locked block sections is to meet the train emergency braking distance [3]. This mode wastes resources and is inefficient for slow trains, because trains in practice do not run at the same speed, especially mixed trains running same line and trains equipped with different types of control systems and degraded equipment. The reason why this mode has still been applied now includes two aspects. One reason is standardization of operation to prevent human errors. The other is that the train route controller (station dispatchers or station self-discipline machine (SSDM) in CTC) cannot get the train speed and braking ratio information. However, as the communication, computer, and control technology are applied continually in railway signalling system, wireless communication technology in particular solves the problem of bidirectional data transmission between train and ground equipment. The station dispatchers and CTC equipment can receive the train speed and braking ratio in real-time, which allows us to realize a variable train-approach locking section. 
This paper proposes a novel method to calculate the distance of a train-approach locking section dynamically based on train speed value, braking parameters, and the level of train control system, in order to realize a variable train-approach locking section. This method can modify the receiving route and reduce the time that a station is occupied by dynamically controlling train operation. This results in an improved passing capacity for stations.

Harrod [4] addresses timetabling models by their structure and capabilities rather than their prior application. Four models are discussed according to whether they explicitly model the track structure, and whether the timetable is intended to be periodic or aperiodic, including Mixed Integer Sequencing Linear Program (MISLP), Binary Integer Occupancy Program (BIOP), Hypergraph Formulation, and Periodic Event Scheduling Program (PESP). MISLP is appropriate for real valued scheduling of single period schedules. However, it is limited to pairwise train dispatching decisions, the starting data set must be known feasible, and it does not provide any direct analysis of the track infrastructure. BIOP is especially suited for the combinatorial packing of train paths within a complex network or the economic valuation of a segment of track. The hypergraph model is capable of explicitly representing the track structure and self-selecting the solution set of trains in the same manner as the BIOP model, and thus it may replace and supersede the BIOP model in many relevant applications. PESP is ideally suited for the solution of periodic timetables with many interrelated passenger connections between trains. However, it shares the characteristic of MISLP in that it cannot resolve data sets where a feasible timetable does not exist.

Dingler et al. [5] intend to use new technologies including Communications-Based Train Control (CBTC) and Electronically Controlled Pneumatic (ECP) brakes to improve railroad capacity and operating efficiency. The potential impact of each element was assessed and compared with the various baseline conditions and conventional technologies to understand the incremental effect. These results will tend to be route and network specific, so individual railroads will need to conduct these analyses to understand the effects on their own systems.

$\mathrm{Li}$ and Tang [6] give an idea that the heterogeneity of signalling systems used in a railway line usually reduces capacity. Blocking time model was used to exposit the headway of signalling systems as well as the headway between trains which are running in different signalling systems. To analyze the effect on capacity when trains are in different signalling systems sharing a line of Beijing metro, they use the simulation software Railsys to solve it. Finally, they evaluated the effect of relationship between capacity and the mixture and sequence of trains as well. These analyses attempt to provide a better understanding of how the signalling systems affect railway capacity and a complete understanding of one factor on capacity. Furthermore, the results can help planners verify if the urban transit lines satisfy the capacity demand and adjust timetable arrangement.

Rudjanakanoknad et al. [7] use the time-space diagram analysis technique to create models comparing single and double-track railway system capacities, with the empirical data from State Railway of Thailand. They show graphical illustration to determine the relationships among operating variables using a set of simple equations. However, some limitations exist: some variables that affect operations such as slowdown when moving to side tracks were excluded. Second, the number of side tracks may not be located in equidistance configuration; this would affect capacities. Also, this research did not take the stochastic effects of train speeds, waiting times at stations, and so forth, in the calculations.

Talebian and Zou [8] develop a hypergraph-based, twolevel approach to sequentially minimize passenger and freight costs while scheduling train services for high performance passenger and freight train operations on shared-use corridors in the US. At the upper level, they identify passenger train schedules that minimize passenger side cost, while at the lower level they develop freight train schedules that minimize freight side cost. Using realistic parameter values, the numeric experiments show that passenger cost due to schedule delay is comparable to in-vehicle travel time cost for short-distance travel.

Schlake [9] has an idea that the effectiveness and efficiency of railcar inspection is critical to freight railroad operations, as it directly affects safety, reliability, mainline efficiency, and terminal performance. Through improved railcar condition monitoring, these technologies have the potential to reduce equipment-caused derailments and inservice failures (ISFs), train delays and variability in mainline operations, and operational waste in railroad terminals. The author applied lean manufacturing methods to the railcar maintenance process, quantifying both direct and indirect waste. The results of these analyses indicate that the costsavings from improved mainline and terminal efficiency through the use of automated condition monitoring technologies (ACMT) are comparable in scale to the costsavings from the potential reduction in equipment-caused derailments.

In order to analyze effects of different signalling systems on train performance in terms of energy consumption and travel times, Corapi et al. [10] propose a model framework for providing optimal driving strategies and related speed profiles which minimize the energy consumption of rail convoys. They propose a simulation-based approach for evaluating rail operating costs under different signalling systems by implementing energy-efficient strategies. The proposed method was applied in the case of the Cumana railway; the result has shown the effects of the different signalling systems on user and train operator costs.

Burdett and Bevrani [11] propose an approach which integrated track duplication and section subdivisions for measuring and planning railway capacity expansion activities. Track duplications are quite costly and permanent and construction times may also be prohibitive. The track subdivision approach utilizes a profile of the traveling time and gradient and/or velocity on all parts of one section if it is available. This approach provides a valuable reference point to compare other approaches, for example, those for determining operational capacity. The results of simulation activities can also be compared to this reference point. 
Abril et al. [12] and Wang et al. [2] analyzed factors which affect the capacity of the railway, including train running speed, train stops mode in station, heterogeneity of traffic flow, distance at which signals are placed, and robustness of the train schedule. UIC406 [13] proposes a method to assess the practical available capacity by compressing buffer time between running line in train diagram. Landex [14-16] proposed that the consumption of railway capacity can be assessed using a method of train diagram compression, and capacity utilization rate can be expressed by the number of trains, the average speed, and the heterogeneity and stability of the train diagram. Harrod [17] evaluated the capacity factors of a mixed speed railway network within a linear, discrete time network model that maximizes an objective function of train volume, delays, and idle train time. He proposed some practical recommendations of train scheduling. Quaglietta et al. [18] introduce one of the outputs of the European project ON-TIME: a framework for the automatic real-time management of railway traffic, designed for being standard and interoperable across different European railways. The aim is to make use of mathematical algorithms to maintain the required service availability during unplanned disturbances to operations. Existing research mainly concentrates on station capacity analysis and calculation; this paper studies how to improve station passing capacity.

\section{Hardware Structure of System}

The method proposed in the manuscript is based on the station equipment to know the speed and position of the train dynamically. It is necessary to set up bidirectional wireless channel between station and train. The on-board equipment can transmit the train speed and position to station equipment. This method can be applied to all signalling systems in which a wireless communication device is installed.

Some additional hardware devices must be added to the CTC system and on-board equipment in order to realize implementation of a variable train-approach locking section, as shown in Figure 1.

CTC system consists of three parts: the dispatching center subsystem, the station subsystem, and the network subsystem, which operates between the dispatching center and stations. Several Application Servers (AS), one train data collector (TDC), and a dispatching command and dispatching telephone (DC\&DT) are in the dispatching center. The station self-discipline machine (SSDM), manmachine interface (MMI), and interface to 6502 interlocking or computer based interlocking (6502 or CBI), and train number are components of the station subsystem [19].

The added equipment is one application server ( $\mathrm{ASi}$ ) in each station, several data acquisition units (DAU) with interface to TAX2 on board. The DAU was used to gather information on train number, speed, location, braking ratio, train type, and control level which were transformed to ASiby wireless transmission. The length of approach locking section was calculated by the ASi and provided the basis for train route setting.
Train number is a significant parameter for train tracking and route controlling for CTC. The train number is its unique ID which is distinguished and tracked by CTC and is further checked and confirmed by the train number system. All of these systems are in order to avoid confusion and to avoid security issues.

\section{A Calculating Model for the Length of a Variable Train-Approaching Locking Section}

China Train Control System (CTCS) is divided into five levels, namely, CTCS- $n$. Level 0 is for the line below $120 \mathrm{~km} / \mathrm{h}$, Level 1 is for the line below $160 \mathrm{~km} / \mathrm{h}$, Level 2 is for the line within $160 \sim 250 \mathrm{~km} / \mathrm{h}$, Level 3 is for the line within $250 \sim 350 \mathrm{~km} / \mathrm{h}$, and Level 4 is designing for the line higher $350 \mathrm{~km} / \mathrm{h}$. Level $n$ refers to the corresponding level of the train control system. CTCS-2 and CTCS-3 are used to control train operation in high-speed railway.

The method proposed in the manuscript can be used for both high-speed railway operations and general speed railway operation. This method is more effective for highspeed railway than for general speed railway. This application of the method in high-speed railways is an example of this manuscript. This method can be applied to all signalling systems in which a wireless communication device is installed.

Calculating a model of the length of a variable trainapproaching locking section attempts to resolve situations of different train speeds, different braking parameters, and different control levels, based on the design standards of a fixed train-approach locking section. Setup of the timing of the train route is also deduced.

3.1. The Length of Fixed Train-Approaching Locking Section. The length of a fixed approach locking section must meet requirement of TB10621-2009 [3], item 14.5.5, which is as follows: "the length of the approach locking section of ground signals should ensure the vehicle-borne equipment on the maximum speed train can not trigger braking outside the length after shutting down the signals." The length of a fixed train-approach locking section should not be less than full service braking distance with the designed train speed, and the transforming time of the train receiving command as well as the running distance during the equipment action (CTCS3 control system also included the maximum permissible communication outage time) must also be considered. The speed value, braking performance, and control level were constant in the calculation of fixed train-approaching locking section, which meant the maximum speed, the worst braking performance, and the lowest control level should be satisfied; this is called the most unfavorable principles [20]. The train route approaching locking section $L_{j}$ includes the full service braking distance $L_{b}$, the safety protection distance $L_{s}$, the distance that the train runs during the information transfer and the braking trigger $L_{c k}$, and the rounding distance composed of integer block sections of approach locking section $L_{r 1}$, as shown in Figure 2.

$$
L_{j}=L_{r 1}+L_{c k}+L_{b}+L_{s} \text {. }
$$




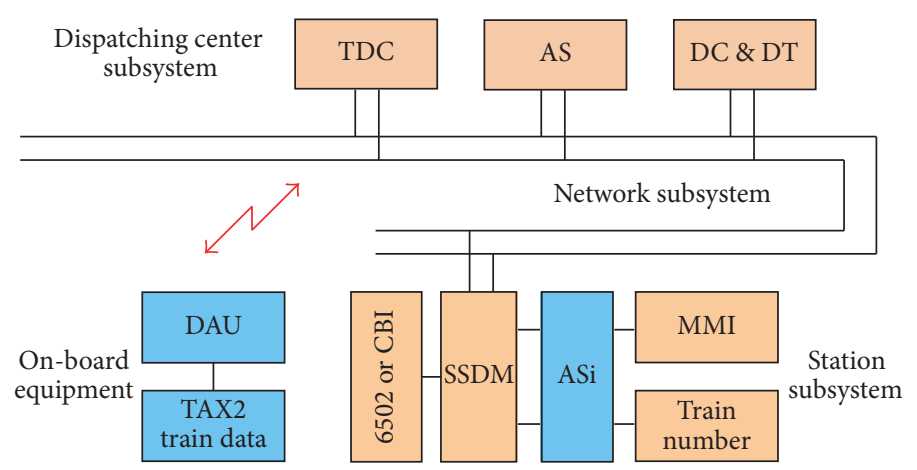

FIGURE 1: The structure of systems in CTC.

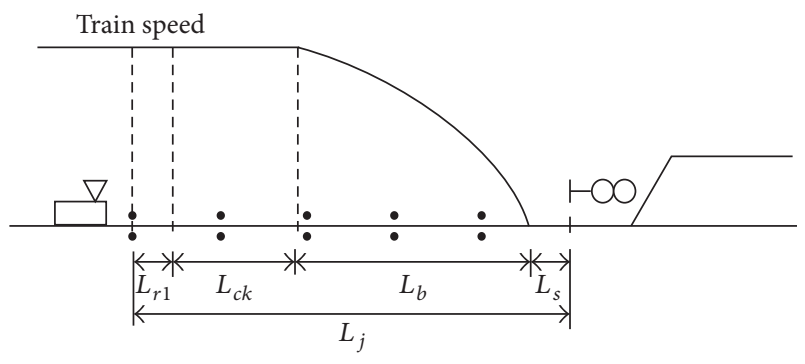

FIGURE 2: The composition of train route approach locking section.

The length of fixed train-approach locking sections could be calculated, according to computation of train traction calculation $[21,22]$ :

$$
\begin{aligned}
L_{j}= & L_{r 1}+\frac{v_{\max } \cdot\left(t_{c}+t_{k}\right)}{3.6} \\
& +\sum_{v_{j}=v}^{v_{j+1}=0} \frac{1000 \cdot(1+r) \cdot\left(v_{j}^{2}-v_{j+1}^{2}\right)}{25.92 \cdot g \cdot c_{b}}+L_{s},
\end{aligned}
$$

where $L_{j}$ is train route approaching locking section, m. $v_{\max }$ is the maximum speed value of the train, $\mathrm{km} / \mathrm{h} . t_{c}$ is the transformation time of the braking command received by the train and the triggering time of service braking by the vehicleborne equipment, s. $t_{k}$ is the idling braking time, s. $v_{j}, v_{j+1}$ are the start speed and the terminal speed during the interval of braking speed, $\mathrm{km} / \mathrm{h} . r$ is rotational mass coefficient that was the ratio between the train rotational mass and total mass. $g$ is acceleration of gravity, $9.81 \mathrm{~m} / \mathrm{s}^{2} . c_{b}$ is the mean value of service braking resultant force in the process of $v$, 0 , in the interval of train braking speed, N/kN. $L_{s}$ is the safety protection distance, m. $L_{r 1}$ is the rounding distance according to block sections, $\mathrm{m}$.

3.2. The Length of Variable Train-Approaching Locking Section. The train speed value, train type, and control level in the calculation of variable approach locking section were not fixed.
The length of variable train-approaching locking section can be calculated by

$$
\begin{aligned}
L_{j x}(v, i, n)= & L_{r 1}+\frac{v \cdot\left(t_{c}(n)+t_{k}(i)\right)}{3.6} \\
& +\sum_{v_{j}=v}^{v_{j+1}=0} \frac{1000 \cdot(1+r) \cdot\left(v_{j}^{2}-v_{j+1}^{2}\right)}{25.92 \cdot g \cdot c\left(v_{j}, i\right)}+L_{s},
\end{aligned}
$$

where $v$ is the initial speed value of the train, $\mathrm{km} / \mathrm{h} . t_{c}(n)$ is the transforming time of braking command received by the train under the level of $n$, s. $t_{k}(i)$ is the idling braking time of $i$ type train, s. $c\left(v_{j}, i\right)$ is the mean value of service braking resultant force of $i$ type train in the process of $v_{j}, v_{j+1}, \mathrm{~N} / \mathrm{kN}$.

3.3. Timing for Setting Train Route. The process of route building requires a certain time, so the timing to set route should not be too early or too late. To arrange it too early is a waste of station resources and to arrange it too late is a threat to train safety. Accordingly, the timing of setting a route should match the train speed, location, and route building time. The process of route building is route arranging, station route choice, switch controlling, route interlocking, and signal controlling. The duration of the route building process begins from the command of automatic routing given by the station according to plan to the new movement authority received by the vehicle-borne equipment after the protecting signal opening. CTCS- 2 and CTCS-3 are two different train control systems. Their equipment composition is very different. Because of the discrepancy between CTCS-2 and CTCS- 3 on the information transmitting channel of the vehicle and ground as well as discrepancy introduced by the subsystem for movement authority, the time for setting train routes is different.

3.3.1. Route Building Time in CTCS-2. The equipment taking part in the process of building the train route was CTC, Train Control Center (TCC), CBI, track circuit, balise, switch, signal, and vehicle-borne equipment. The interaction of information in different stages was shown as in Figure 3.

The time of each part in the process of route building (see Figure 3) can be obtained by relevant design specifications. 


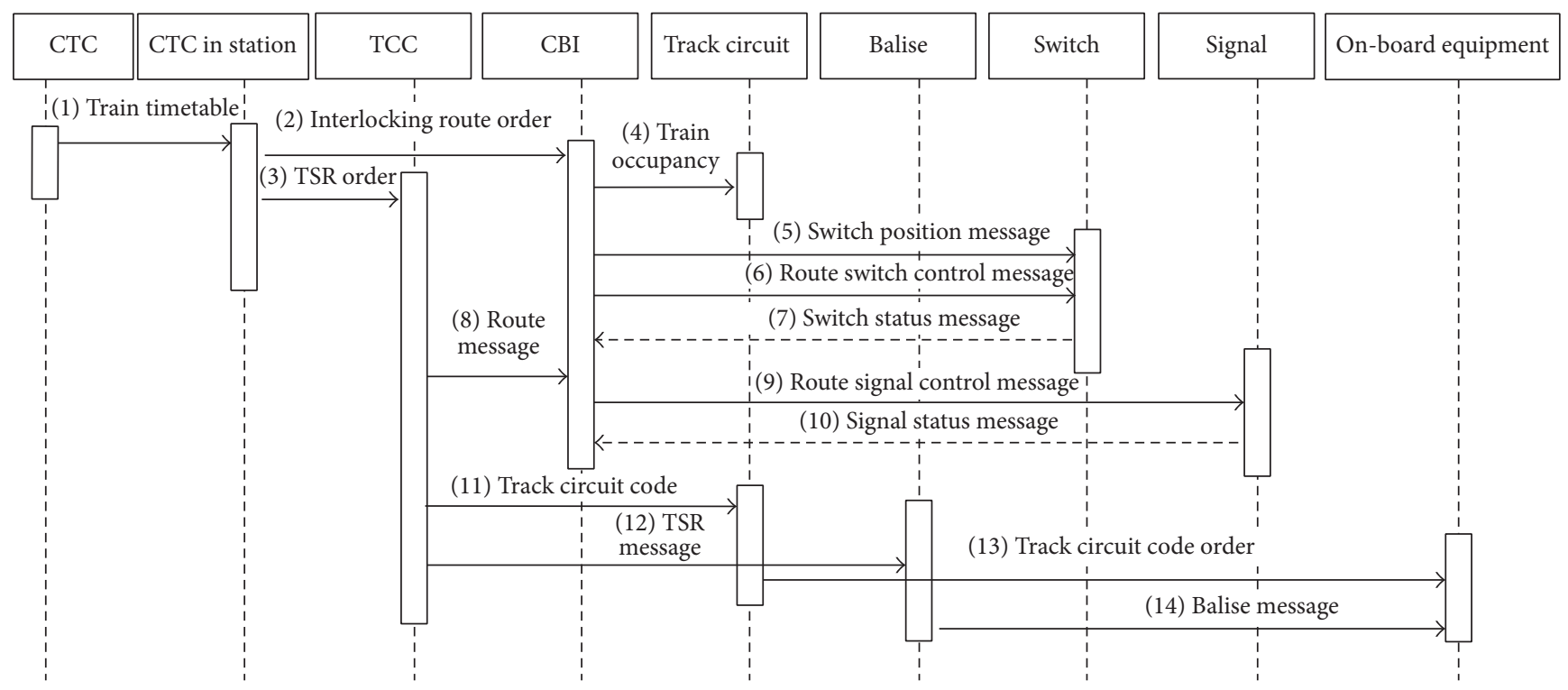

FIGURE 3: Sequence chart of information interaction for building route in CTCS-2.

(1) Because the communication is judged to be interrupted while the CTC and CBI can not receive each other for $3 \mathrm{~s}$, the maximum transfer time between CTC and CBI is $3 \mathrm{~s}$.

(2) The CBI time mainly refers to switch operating time. The actuation time of the ZYJ7 electrohydraulic switch machine is below $17.5 \mathrm{~s}$, which is $6.6 \mathrm{~s}$ by a $\mathrm{S} 700 \mathrm{~K}$ electric switch machine [23]. However, to build a route requires several groups of switches to actuate sequentially. Accordingly, the time of switch operating is

$$
t_{1}(h)=\sum_{i=0}^{h} t_{i}
$$

where $t_{1}(h)$ is total operating time of $h$ groups of switches, s. $t_{i}$ is operating time of $i$ th switch, s.

(3) The transmitting time for data between CBI and TCC was $200 \mathrm{~ms} \sim 500 \mathrm{~ms}$, and the communication was judged as interrupted while the CBI and TCC can not receive each other for $6 \mathrm{~s}$, so the transmitting time between CBI and TCC is $6 \mathrm{~s}$.

(4) The processing time of TCC was generally $0 \sim 1 \mathrm{~s}$, so $1 \mathrm{~s}$ is chosen.

(5) The information transformed from TCC to vehicleborne equipment was generally $1 \sim 4 \mathrm{~s}$, so $4 \mathrm{~s}$ is chosen.

(6) The time from receiving the new information by vehicle-borne equipment to displaying was below $3.6 \mathrm{~s}$, so $3.6 \mathrm{~s}$ was chosen as processing time by vehicle-borne equipment.

So, the time to build a route under the application of CTCS- 2 is

$$
t_{p}(2, h)=17.6+t_{1}(h) .
$$

3.3.2. Route Building Time in CTCS-3. The interaction of information in CTCS-3 is shown in Figure 4.

Similar to CTCS-2, the time required by all equipment is as follows.
(1) The transfer time from CTC to CBI is chosen as $3 \mathrm{~s}$, which was same as interruption time.

(2) The principle of CBI time is the same as CTCS-2, so the time of switch operating is also $t_{1}(h)=\sum_{i=0}^{h} t_{i}$.

(3) The $\mathrm{RBC}$ receives the route information from the CBI, so the transmitting time between $\mathrm{CBI}$ and $\mathrm{RBC}$ is $3 \mathrm{~s}$.

(4) The processing time of RBC was generally $0.4 \sim 0.8 \mathrm{~s}$, so $0.8 \mathrm{~s}$ is chosen.

(5) The permitted time for communication interruption between vehicle-borne equipment and $\mathrm{RBC}$ is $7 \sim 20 \mathrm{~s}$, so the time of movement authority transformation from RBC to vehicle-borne equipment by the GSM-R is chosen to be $20 \mathrm{~s}$.

(6) The time from receiving the new information by vehicle-borne equipment to displaying it should never be more than $2 \mathrm{~s}$, so $2 \mathrm{~s}$ was chosen.

So, the time $t_{p}$ to build a route under the application of CTCS-3 is

$$
t_{p}(3, h)=28.8+t_{1}(h) .
$$

3.4. Model of Triggering Time for Receiving Route. The triggering time for receiving route is beginning to set the route. If it is set too early, the track and switches can not be used by other trains, which reduces efficiency. But if it is set too late, braking will be triggered. The best timing is when the train takes up the first approach locking section; the route is just built.

The station needs to set receiving route at a lead time. Calculating the trigger timing requires information on the time duration of the train running in the approach locking section and time required to build the route. Based on the regulations of railway technical operation, combined with 


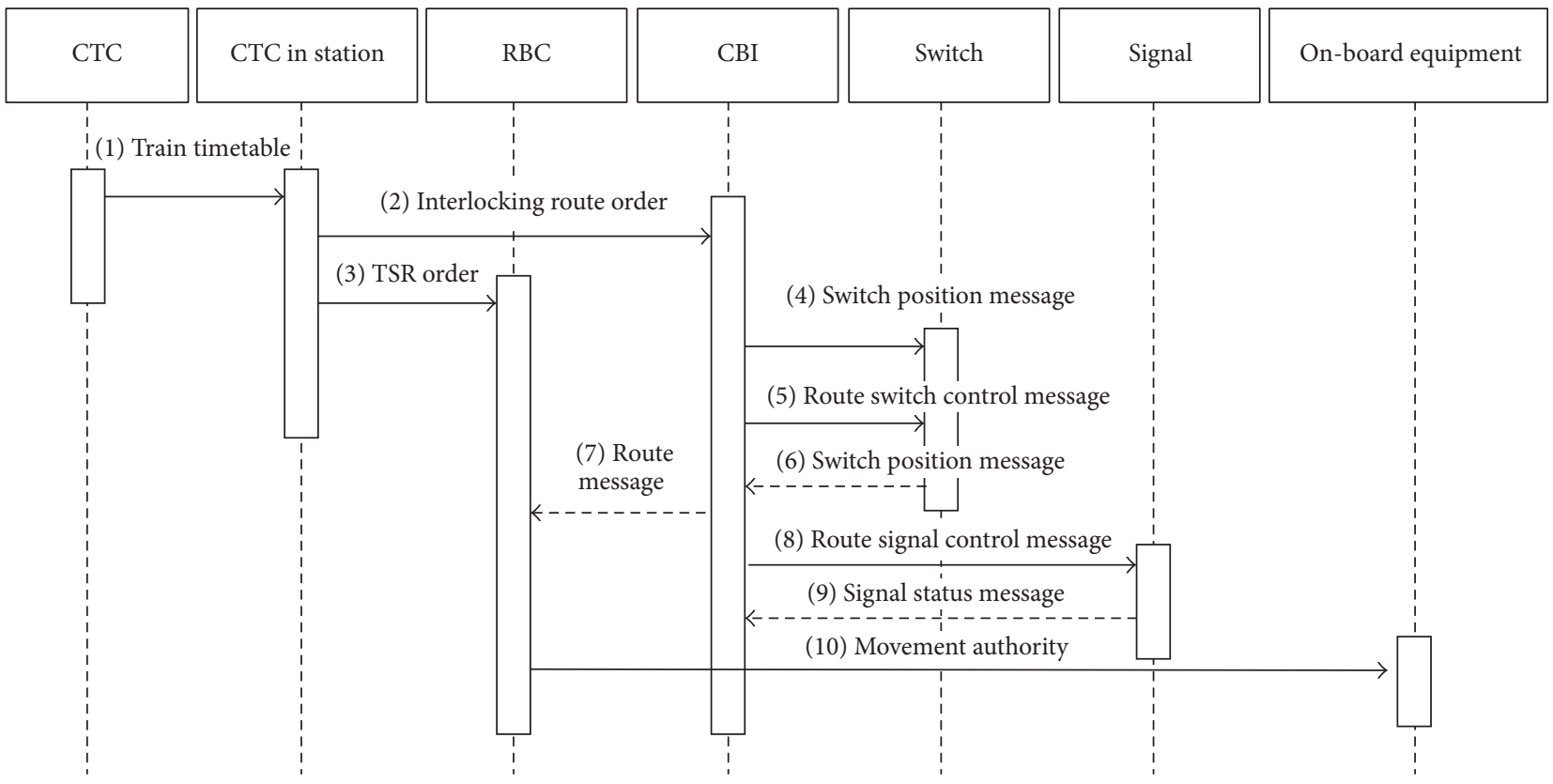

FIGURE 4: Sequence chart of information interaction for route building in CTCS-3.

practical line parameters, design speed, and station layout, the triggering time can be calculated.

The triggering method for the receiving route can be divided into two types: time-triggered and space triggered. Time-triggered mode is based on the estimated arrival time and the CTC of the station triggering the routing command automatically (as specified by regulations). Space triggered mode means that the CTC of the station triggers the command to route automatically when the train arrives at the required position. The station should trigger the command for routing $t_{c}$ before the train's arrival, according to timetriggered mode:

$$
t_{c} \geq t_{j x}+t_{p}
$$

where $t_{j x}$ is the running time of the train in the variable trainapproaching locking section, $s . t_{p}$ is the time of the route building by the station, $\mathrm{s}$.

The real-time speed can affect the distance of approach locking section and the value of $t_{j x}$. The variable approach locking section is composed of four parts from (3), and $t_{j x}$ can be obtained by each part. is

Similarly, the distance calculated by space triggered mode

$$
L_{c} \geq L_{j x}+L_{b z}
$$

where $L_{c}$ is the distance of handling the route triggered, which is made up of an integer number of block sections, m. $L_{b z}$ is the prepared distance, which is the sum of the distance of the train running during the time taken by the station handling of the route and the distance of secondary rounding, consisting of an integer number of block sections; $L_{b z}=L_{b}+L_{r 2}$, m. $L_{r 2}$ is secondary rounding, $\mathrm{m}$.

\section{Analysis of Improving the Passing Capacity of Station}

4.1. Concept of Passing Capacity. Passing capacity of a station in a high-speed railway is the number of trains received by and departing from the station in a nychthemeron by a logical organization program, within limits of existing equipment capabilities. There are many factors that influence the capacity, such as station equipment, speeds level of the train, quantity of trains, and the practical level of organization [24]. The current methods of calculating the passing capacity of station utilize ratio calculation, graphic calculation, analytical calculations, computer simulation, and queuing theory. The foundational principle of these methods is to obtain the passing capacity by analyzing the time used at throat points (the points in throat area at both ends of station) by all types of station operations, occupancy time of arrival-departure track time, and the coefficient of unused line.

The regulation of a station's occupancy time and arrivaldeparture tracks of high-speed railway station is different from that of medium-speed railway station, because there are no trains running on the line at night. According to high-speed railway design specifications, the passing capacity of high-speed railway station has unique characteristics, the value of which needs to be calculated by peak hour operation in a nychthemeron.

4.2. Method. The organization form of a high-speed railway is different from existing lines, so the method of passing capacity calculation is also unique.

(1) There is no need to consider the freight operation time for calculation on a high-speed railway, because 
the high-speed railway only handles the passenger transport.

(2) Because of integration, calculation does not include the train pick hang and uniting procedures of the motor train unit.

(3) The time period chosen needs to refer to train operation during peak hours in a day and night period, based on features of the station.

(4) The occupancy time standard of a throat point by practical station operation and arrival-departure track needs to be revised as appropriate.

(5) The fixed operation items should also be revised according to the practice of the station.

From these considerations, the total calculation procedures of passing capacity by utilization ratio are as follows.

Step 1. Analyze the practical data of the chosen station, which includes static data and dynamic data.

(1) Analysis of the static data:

(i) Obtain the number of arrival-departure tracks and layout of throat point of station by plane layout of signals and group the throat point.

(ii) Define the variety of station operations, which include starting, occupancy, turn-back, passing, receiving, and departure.

(2) Analysis of the dynamic data:

(i) Gather statistics of the quantity of train to be arranged in a nychthemeron or calculation period.

(ii) Classify the statistics according to operation characteristics.

Step 2. Determine the time standard of occupancy of station equipment, which includes time standards of throat point and the arrival-departure track.

(1) Define the time standard of occupancy of throat points.

The time standard of occupancy of throat point $t_{i j}$ is

$$
t_{i j}=t_{i 1}+t_{i 2}
$$

where $t_{i 1}$ is the time taken by the train running during approach to the locking section and handling time by the station: $t_{i 1}=t_{j x}+t_{p}$, min. $t_{i 2}$ is the time from the train passing the arrival signal to the throat point route unlocking, min.

The standard time of occupancy of throat points by starting operation is

$$
t_{i f}=t_{i 3}+t_{i 4}
$$

where $t_{i 3}$ is the time to build the starting route by station, min. $t_{i 4}$ is the time taken from the route to be built to the throat point route unlocking, $\min$.
(2) Define the occupancy time standard of the arrivaldeparture track.

The standard time of occupancy of the arrival-departure $\operatorname{track} t_{j f}$ is

$$
t_{j f}=t_{i j}+t_{s}+t_{i f}
$$

where $t_{i j}$ is the time from handling a route by a station to stopping at the arrival-departure track by the train, min. $t_{s}$ is the time duration of train stopping, min. $t_{\text {if }}$ is the time from the train pulling out of the arrival-departure track to the arrival-departure track unlocking, min.

Step 3. Calculate the occupancy time of throat points and the arrival-departure track. This is the time standard of the throat point and the arrival-departure track defined in Step 2, and then obtain the total occupancy time of all operations in a nychthemeron or specified period with the data of Step 1.

The total occupancy time of all throat points is $t_{y 1}, t_{y 2}, \ldots, t_{y n}$, so $t_{y}=\max \left\{t_{y 1}, t_{y 2}, \ldots, t_{y n}\right\}$ if the station had $M$ arrival-departure tracks and the total occupancy time of each arrival-departure track is $t_{x 1}, t_{x 2}, \ldots, t_{x M}$. The total occupancy time of all arrival-departure tracks is $t_{x}=\sum_{i=1}^{M} t_{x i}$.

Step 4. According to the resulting time obtained in Step 3 as well as the utilization ratio formula, the passing capacity of a station could be calculated [25].

The practical utilization ratio of passing capacity of the throat points $K$ is

$$
K=\frac{t_{y}-\sum t_{g}}{\left(T M-\sum t_{g}\right)(1-\alpha)},
$$

where $\sum t_{g}$ is the total occupancy time of throat point of fixed operation in the given time, min. $\alpha$ is the coefficient of line leisure of throat point. $T$ is the period of time chosen according to analysis characteristics, min. So the passing capacity of throat point to all directions is

$$
\begin{aligned}
& N d_{j}=\frac{n d_{j}}{K}, \\
& N d_{f}=\frac{n d_{f}}{K},
\end{aligned}
$$

where $N d_{j}, N d_{f}$ are the capacity of receiving-departure of $d$ direction. $n d_{j}, n d_{f}$ are the number of receiving-departure of $d$ direction.

The practical utilization ratio of passing capacity of the arrival-departure track $K$ is

$$
K=\frac{t_{x}-\sum t_{g}}{\left(T M-\sum t_{g}\right)(1-\alpha)} .
$$

So the capacity of receiving-departure of all directions $N d$ is

$$
N d=\frac{n d}{K} .
$$




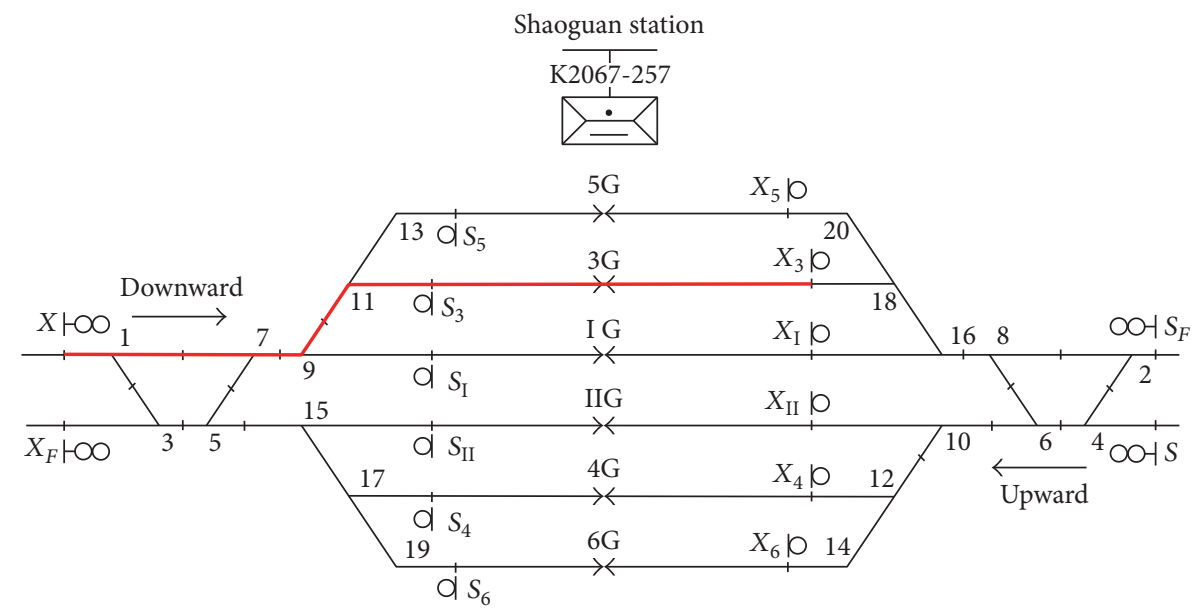

FIGURE 5: The plane layout of signals of Shaoguan station.

TABLE 1: The length of downward positive block section of Shaoguan station.

\begin{tabular}{lccccc}
\hline Block section & L1 & L2 & L3 & L4 & L5 \\
Length (m) & 1663 & 1279 & 1818 & 1801 & 1832 \\
Block section & L6 & L7 & L8 & L9 & L10 \\
Length (m) & 1860 & 1823 & 1874 & 1788 & 1810 \\
Block section & L11 & L12 & L13 & L14 & L15 \\
Length (m) & 1852 & 1850 & 1876 & 1828 & 1948 \\
\hline
\end{tabular}

\section{Simulation and Verification}

Taking the practical data of Shaoguan station as an example, the influences of passing capacity by both fixed approach section and variable approach section were comparatively analyzed, and the improvement was calculated. The Shaoguan station is the main station on the high-speed railway line of Beijing to Guangzhou and Shaoguan is a town in the north of Guangdong province in China.

The plane layout of signals of Shaoguan station and length of downward positive block sections are shown as Figure 5 and Table 1, respectively. There are 26 trains of 160 $250 \mathrm{~km} /$ hand 93 trains of $250 \sim 350 \mathrm{~km} / \mathrm{h}$ through Shaoguan station one day, and the type of CTC is FZk-CTC. From Figure 5, the number of throat points is small, and switch transaction reflects in the route of the arrival-departure track. So the passing capacity of Shaoguan station can be expressed by the capacity of arrival-departure track, and the influence by the throat section is very small [26].

5.1. Variable Approach Locking Section. In this study, the design speed of Beijing-Guangzhou high-speed railway is $350 \mathrm{~km} / \mathrm{h}$, and the control system at the time of study was CTCS-3. The types of motor train unit included CRH3C, CRH2C, CRH380, and CRH380B. All of the motor train units passing Shaoguan station were considered to be CRH3C, the rotary mass coefficient of which is $r=0.1, t_{c}=26.8 \mathrm{~s}$, and $t_{k}=1.5 \mathrm{~s}[27,28]$; the relationship between braking deceleration and velocity $v$ is showed in Table 2 .
TABLE 2: The relationship between $a$ and $v$.

\begin{tabular}{lc}
\hline$a$ & $v$ \\
\hline$a=1$ & $0<v \leq 160$ \\
$a=-0.25 v+5$ & $160<v \leq 172$ \\
$a=-0.002 v+1.06$ & $172<v \leq 210$ \\
$a=-0.0034 v+0.7105$ & $210<v \leq 350$ \\
\hline
\end{tabular}

According to (3) and (4), the decreasing length of speed was chosen to be 1 , and the distance $L_{c k}$ of trains running at different speeds in the time of information transformation and the active braking distance $L_{b}$ can be obtained, with data shown in Table 3.

From Table 1, the length of an integral number of block sections $L_{j x}$ can be obtained, the value of which was the minimum of the sum of $L_{c k}, L_{b}$, and $L_{s}$. The rounding distance $L_{r l}$ can also be obtained.

To sum up, under the control of CTCS- 3 the information on the length and time of the downward variable approach section $L_{j x}$ as it changes with speed can be seen in Table 3 . Similarly, the length of upward moving variable approach sections can also be obtained.

5.2. The Triggering Time. The approach locking section (upward moving and downward moving arrival signals) of Shaoguan station is fixed with 8 approaches. The latest route handling time is the occupancy moment of the train. Based on the principle of using the latest time to trigger the route, the timing of the latest handling route for fixed approach locking sections can be calculated by spatial distance.

From Figure 5, the amount of switches in downward 3G receiving route is 4 , so the time for switch $(1,7,9$, and 11) transaction is $t_{1}(h)=\sum_{i=0}^{h} t_{i}=17.5 \times 4=70 \mathrm{~s}$.

From (6), the time to build a route under the control of CTCS -3 is $t_{p}(3, h)=28.8+t_{1}(h)=28.8+70=98.8 \mathrm{~s}$; the distance of the train running in the time $L_{p}$ and distance of secondary rounding $L_{r 2}$ can be calculated. According to 
TABLE 3: The downward variable approach locking section of Shaoguan station.

\begin{tabular}{lcccccc}
\hline $\begin{array}{l}\text { Speed } \\
v(\mathrm{~km} / \mathrm{h})\end{array}$ & $\begin{array}{c}\text { Active braking } \\
\text { distance } L_{b} \\
(\mathrm{~m})\end{array}$ & $\begin{array}{c}\text { Braking } \\
\text { transmission } \\
\text { distance } L_{c k}(\mathrm{~m})\end{array}$ & $\begin{array}{c}\text { Variable additional distance }(\mathrm{m}) \\
\text { Safety protection } \\
\text { distance } L_{s}(\mathrm{~m})\end{array}$ & $\begin{array}{c}\text { Variable approach } \\
\text { Rounding } \\
\text { distance } L_{r 1}(\mathrm{~m})\end{array}$ & $\begin{array}{c}\text { The number of block sections that } \\
\text { distance } L_{j x}(\mathrm{~m})\end{array}$ & $\begin{array}{c}\text { compose the variable approach } \\
\text { locking section distance }\end{array}$ \\
\hline 160 & 1086.42 & 1257.78 & 110.00 & 484.8 & 2939 & 2 \\
180 & 1464.01 & 1415.00 & 110.00 & 1767.99 & 4757 & 3 \\
220 & 2524.05 & 1729.44 & 110.00 & 393.51 & 4757 & 3 \\
250 & 3506.49 & 1965.28 & 110.00 & 976.23 & 6558 & 5 \\
300 & 5479.05 & 2358.33 & 110.00 & 442.62 & 8390 & 6 \\
320 & 6391.18 & 2515.56 & 110.00 & 1233.26 & 10250 & 7 \\
350 & 7897.96 & 2751.39 & 110.00 & 1313.65 & 12073 & 7 \\
\hline
\end{tabular}

TABLE 4: The space triggering time of downward positive route for receiving trains of Shaoguan station.

\begin{tabular}{lcccc}
\hline $\begin{array}{l}\text { Train speed } \\
v(\mathrm{~km} / \mathrm{h})\end{array}$ & $\begin{array}{c}\text { The length of } \\
\text { fixed approach } \\
\text { section }\end{array}$ & $\begin{array}{c}\text { The length of } \\
\text { variable approach } \\
\text { section }\end{array}$ & $\begin{array}{c}\text { Trigger time for fixed } \\
\text { approach section (the } \\
\text { corresponding number } \\
\text { of blocking sections) }\end{array}$ & $\begin{array}{c}\text { Trigger time for variable } \\
\text { approach section (the } \\
\text { corresponding number of } \\
\text { blocking sections) }\end{array}$ \\
\hline $\begin{array}{l}230 \sim 269 \\
270\end{array}$ & 8 & 4 & 15 & 8 \\
$271 \sim 307$ & 8 & 4 & 15 & 15 \\
$308 \sim 333$ & 8 & 5 & 15 & 10 \\
$334 \sim 340$ & 8 & 6 & 15 & 15 \\
$341 \sim 350$ & 8 & 6 & 15 & 13 \\
\hline
\end{tabular}

(8), the distance $L_{c}$ of handling the route in advance can be obtained from Table 4.

5.3. Comparison of Passing Capacity. The Shaoguan station handled 119 arrival-departure trains in total $(n=119)$, with downward moving group being 50 and upward moving group being 69. The stop time of the downward moving group was $116 \mathrm{~min}$, and the upward moving group was $206 \mathrm{~min}$, which is disproportionate. The operation rush-hour time of the high-speed railway was from 7:00 to 23:00 ( $T=960 \mathrm{~min})$. The time for fixed operation on this period was 0 ; that is, $\sum t_{g}=0$. The coefficient of unused service time on the passenger special line was $0.25 \sim 0.35$, so $\alpha=0.35$ in this article. The difference in the passing capacity between the fixed and variable approach locking sections is the timing to handle the route, which depends on the $t_{i j}$. That is, $t_{i j}$ corresponding block section lengths are different.

The relation of length of approach locking sections and time of occupancy to arrival-departure lines with the velocity of single-train running downward into Shaoguan station under the CTCS-3 was obtained. The relation curve spacetime-speed for variable approach locking section is shown in Figure 6. According to the survey, in Shaoguan station all arrival operations, the case that train reaches less than $100 \mathrm{~km} / \mathrm{h}$ to the station did rarely emerge. So the speed interval is determined as starting from $100 \mathrm{~km} / \mathrm{h}$.

The time saved via implementation of variable approach locking section in one route in each nychthemeron is shown in Figure 7.

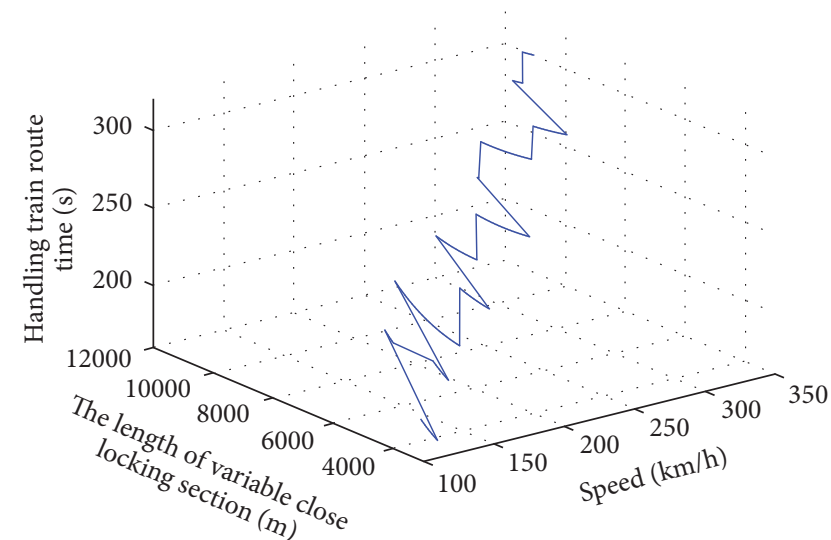

FIGURE 6: The relation curve space-time-speed for variable.

The passing capacity of Shaoguan station is shown in Figure 8 calculated by (15). Although capacity improvement showed a downward trend with the increase in train speed, it is still more efficient than the fixed method. When the train is slow, more capacity is increased. When the train is faster, less capacity is improving.

The factors that influence passing capacity and should be included in the design of throat and arrival-departure tracks are as follows: collinearity at different speeds, imbalance of arrival and departure events, time of maintenance-window, types of motor train units, and the proportion of arrivals (received at the station) to departures. All of the factors have been considered in the example, except the proportion of receiving to departure. 


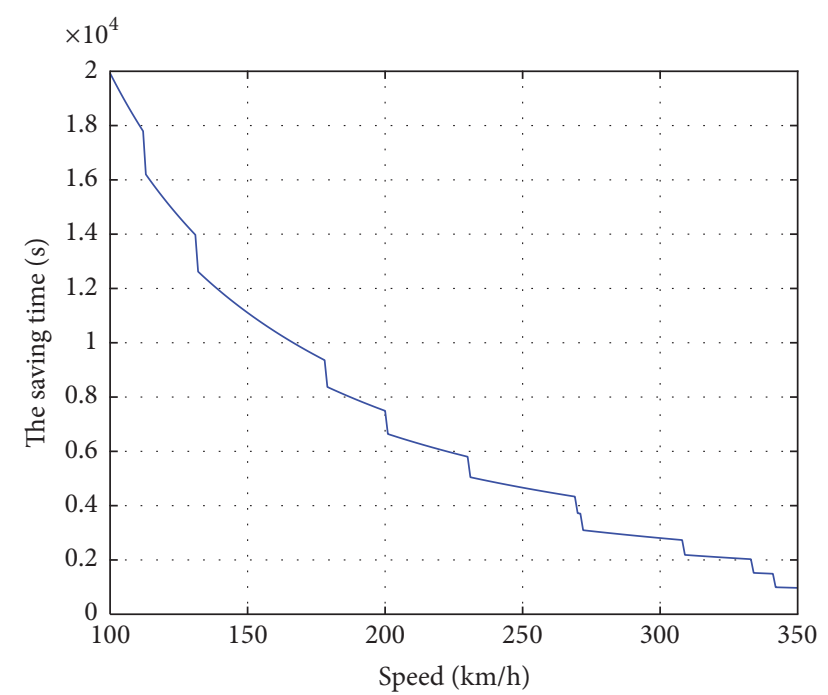

Figure 7: The saving time of one routing of variable approach locking section.

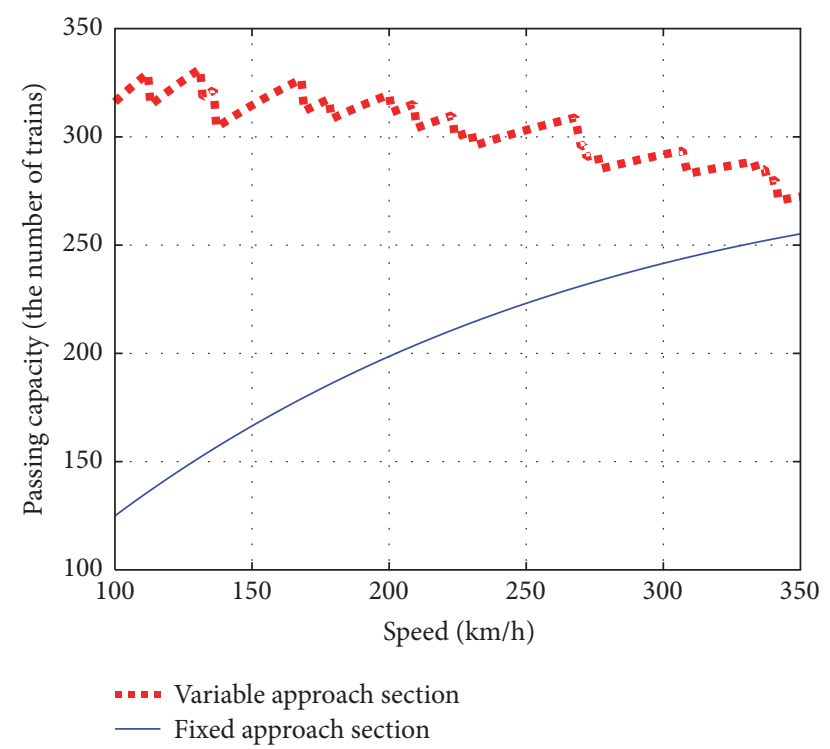

FIGURE 8: The comparison of passing capacity in both conditions.

Types of arrival-departure can be divided into starting, arriving, passing, occupying, and turn-back events. The operations of arrival, passing, and occupying involve handling the route, which in the study was $82.6 \%$ (set $k_{1}=82.6 \%$ ) of the arrival-departure of Shaoguan station.

Abnormal situations, such as equipment failure and construction, caused the train running into the approach section to maintain a low speed, and this was $4.3 \%$ (set $k_{2}=$ $4.3 \%$ ) of the station's receiving-departure events.

The improving passing capacity under the variable and fixed section is

$$
\eta=k_{1} k_{2} \frac{N_{v}-N_{f}}{N_{f}}
$$

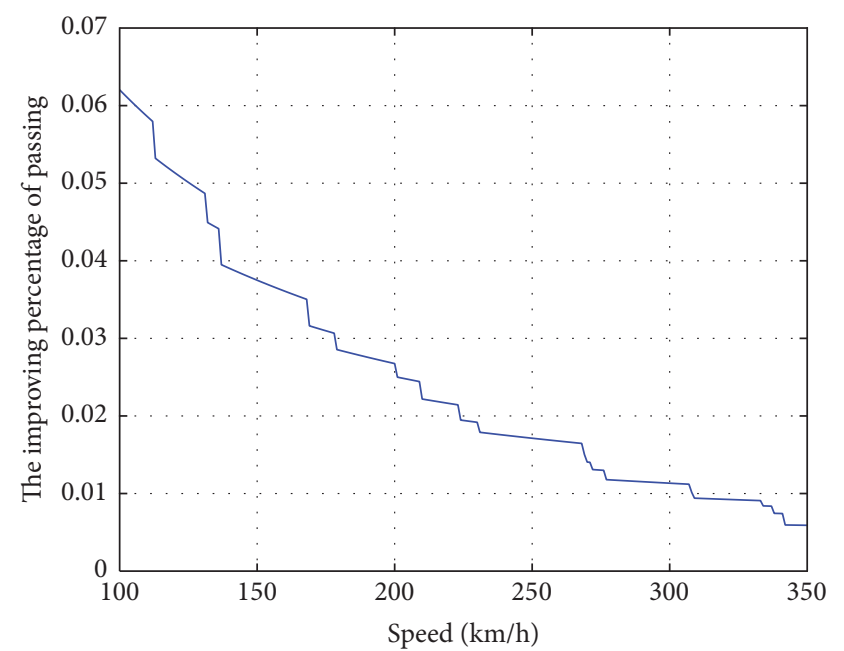

FIGURE 9: The improving percentage of passing.

where $k_{1}$ and $k_{2}$ represent the coefficients, as described above. $N_{v}$ represents the passing capacity under the variable section. $N_{f}$ represents the passing capacity under the fixed section.

By synthesis of the influence factors and the proportionality coefficient of low-speed running, that is, (16), we can obtain the dynamic relationship between improving passing capacity and speed, shown in Figure 9. The descending trend showed improvement at $0.6 \% \sim 6.3 \%$.

\section{Conclusion}

A variable approach locking section was proposed in this paper, and we built the calculation model of variable approach locking section and timing to trigger routing. The influence of the variable approach on passing capacity was studied. In the end, taking Shaoguan station as example, the standpoint that the variable approach locking section could improve the passing capacity was verified, and the improving percentage was $0.6 \% \sim 6.3 \%$.

Comparing with a fixed mode, obviously, variable trainapproaching locking section evenly shortens the train arrival time, which is conducive to restore the delay of train. This capability will be verified in future research.

\section{Competing Interests}

The authors declare that they have no competing interests.

\section{Acknowledgments}

This work was supported in part by the National Natural Science Foundation of China under Grant 61374201 and in part by the State Key Laboratory of Rail Traffic Control and Safety under Grant RCS2016ZZ003. The authors would like to thank Dr. Forrest Fabian Jesse: he revised grammatical and syntactical errors in the manuscript. The authors would like to thank Lingyun Meng, associate professor in State Key Laboratory of Rail Traffic Control and Safety, and Lijiang Dai, 
M.S. degree student, for their contributions. They provided many suggestions related to simulation and verification in this paper. The authors would like to thank KeNiu, doctoral candidate, and HuiMa, M.S. candidate, for their amendments to the manuscript.

\section{References}

[1] Z. X. Zhao, Signalling Control System of Station, People's Traffic Press, Beijing, China, 2013.

[2] X. P. Wang, X. S. Du, W. Hua, and W. T. Yang, "Conditions related to passing capacity of passenger dedicated line stations and its calculation method," Railway Operation Technology, vol. 14, no. 4, pp. 1-3, 2008 (Chinese).

[3] Ministry of Railways of the People's Republic of China, "Code for design of high speed railway,” Tech. Rep. TB10621-2009, 2009 (Chinese).

[4] S. S. Harrod, "A tutorial on fundamental model structures for railway timetable optimization," Surveys in Operations Research and Management Science, vol. 17, no. 2, pp. 85-96, 2012.

[5] M. H. Dingler, Y.-C. Lai, and C. P. L. Barkan, "Effects of communications-based train control and electronically controlled pneumatic brakes on railroad capacity," Transportation Research Record, no. 2159, pp. 77-84, 2010.

[6] W. Li and T. Tang, "The impact of signalling system heterogeneity on urban transit capacity," in Proceedings of the IEEE International Conference on Intelligent Rail Transportation (ICIRT '13), Beijing, China, August 2013.

[7] J. Rudjanakanoknad, P. Deethanasuwan, and P. Wongchaivit, "Time-space diagram models for single-and double-track railway system capacities in Thailand," Journal of Society for Transportation and Traffic Studies, vol. 5, no. 1, pp. 38-49, 2014.

[8] A. Talebian and B. Zou, "Integrated modeling of high performance passenger and freight train planning on shareduse corridors in the US," Transportation Research Part B: Methodological, vol. 82, pp. 114-140, 2015.

[9] B. Schlake, Impact of automated condition monitoring technologies on railroad safety and efficiency [M.S. thesis], University of Illinois at Urbana-Champaign, Champaign, Ill, USA, 2010.

[10] G. Corapi, D. Sanzari, V. Martinis, D. L. Acierno, and B. Montella, "A simulation-based approach for evaluating train operating costs under different signalling systems," WIT Transactions on the Built Environment, vol. 130, pp. 149-161, 2013.

[11] R. L. Burdett and B. Bevrani, "A mathematical framework for expanding a railway's theoretical capacity," in Proceedings of the 17th International Conference on Systems Engineering Modeling and Analysis, vol. 17, pp. 231-234, WASET, Venice, Italy, November 2015.

[12] M. Abril, F. Barber, L. Ingolotti, M. A. Salido, P. Tormos, and A. Lova, "An assessment of railway capacity," Transportation Research Part E: Logistics and Transportation Review, vol. 44, no. 5, pp. 774-806, 2008.

[13] UIC, Capacity (UIC Code 406), International Union of Railways (UIC), Paris, France, 2004.

[14] A. Landex, B. Schittenhelm, A. H. Kaas, and J. Schneider-Tilli, "Capacity measurement with the UIC 406 capacity method," in Proceedings of the 11th International Conference on Computer System Design and Operation in the Railway and Other Transit Systems (COMPRAIL '08), pp. 55-64, Toledo, Spain, September 2008.
[15] A. Landex, "Capacity statement for railways," in Annual Transport Conference at Aalborg University 2007, Aalborg University, Aalborg, Denmark, 2007.

[16] A. Landex, A. H. Kaas, B. Schittenhelm, and J. Schneider-Tilli, "Evaluation of railway capacity," in Proceedings of Trafficdays, Aalborg University, Aalborg, Denmark, 2006.

[17] S. Harrod, "Capacity factors of a mixed speed railway network," Transportation Research Part E: Logistics and Transportation Review, vol. 45, no. 5, pp. 830-841, 2009.

[18] E. Quaglietta, P. Pellegrini, R. M. P. Goverde et al., "The ON-TIME real-time railway traffic management framework: a proof-of-concept using a scalable standardised data communication architecture," Transportation Research Part C: Emerging Technologies, vol. 63, pp. 23-50, 2016.

[19] China Railway Publishing House, Science and Technology of Transportation No. 15, The Distributed Autonomic Centralized Traffic Control System (CTC) Technology Conditions (Interim), China Railway Publishing House, Beijing, China, 2004.

[20] B. H. Mao and X. M. Li, Calculation and Design of Train Operation, People's Traffic Publishing House, Beijing, China, 2013 (Chinese).

[21] H. J. Wei, "Design exploration of train-approach locking section length in high-speed railway," Railway Signalling \& Communication Engineering, vol. 11, no. 1, pp. 26-28, 2014 (Chinese).

[22] Z. Rao, Computation of Train Traction Calculation, China Railway Publishing House, Beijing, China, 2010 (Chinese).

[23] Ministry of Railways of the People's Republic of China, Railway Signalling Maintenance Rules-Technical Standard (TB106212014), 2006 (Chinese).

[24] M. Liu, B.-M. Han, and D.-W. Li, "Calculation and evaluation of carrying capacities at high-speed railway stations," Journal of the China Railway Society, vol. 34, no. 4, pp. 9-15, 2012.

[25] Q. Y. Peng and C. Wen, Transport Organization Foundation of High-Speed Railway, Southwest Jiaotong University Press, Chengdu, China, 2014 (Chinese).

[26] Wuhan-Guangzhou Railway Passenger Dedicated Line Co. LTD, Project Summary of Wuhan-Guangzhou Railway Passenger Dedicated Line, China Railway Publishing House, Beijing, China, 2012 (Chinese).

[27] Technology Department of MOR, CTCS-3 Functional Requirements Specification (FRS) (V1.0), China Railway Publishing House, Beijing, China, 2009 (Chinese).

[28] Technology Department of MOR, CTCS-3 System Requirements Specification (SRS) (V1.0), China Railway Publishing House, Beijing, China, 2009 (Chinese). 


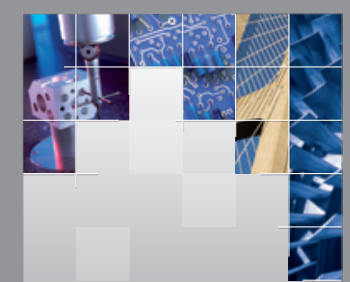

\section{Enfincering}
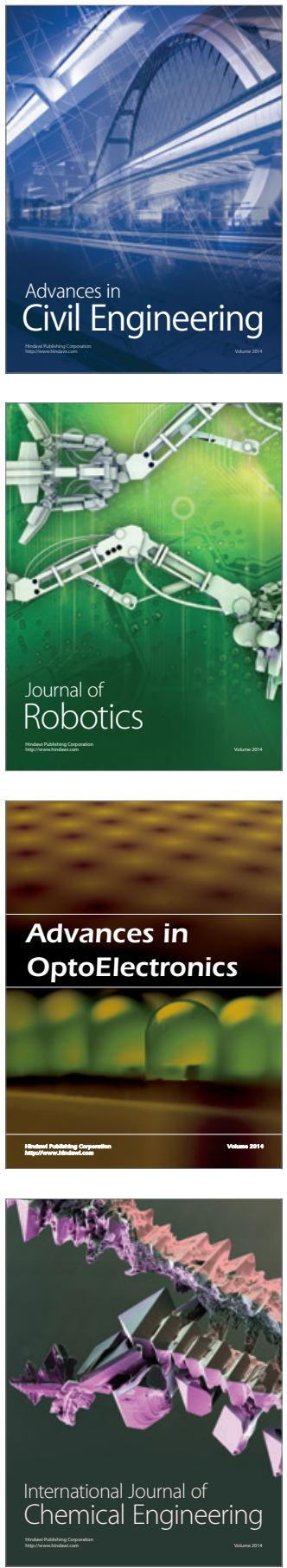

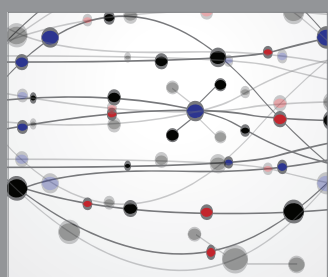

The Scientific World Journal

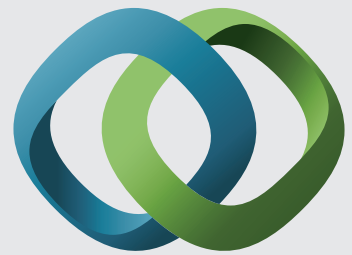

\section{Hindawi}

Submit your manuscripts at

https://www.hindawi.com
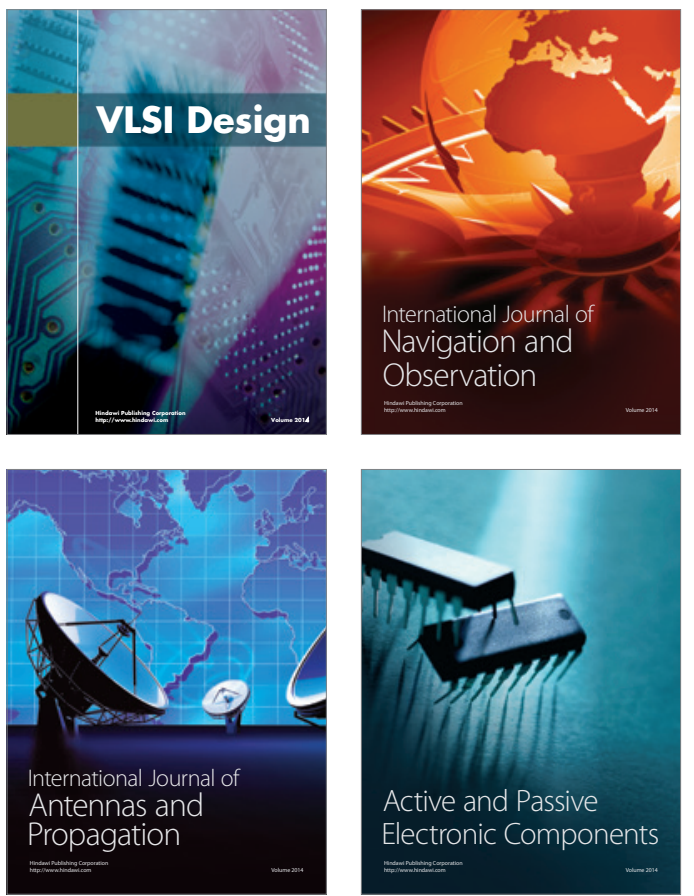
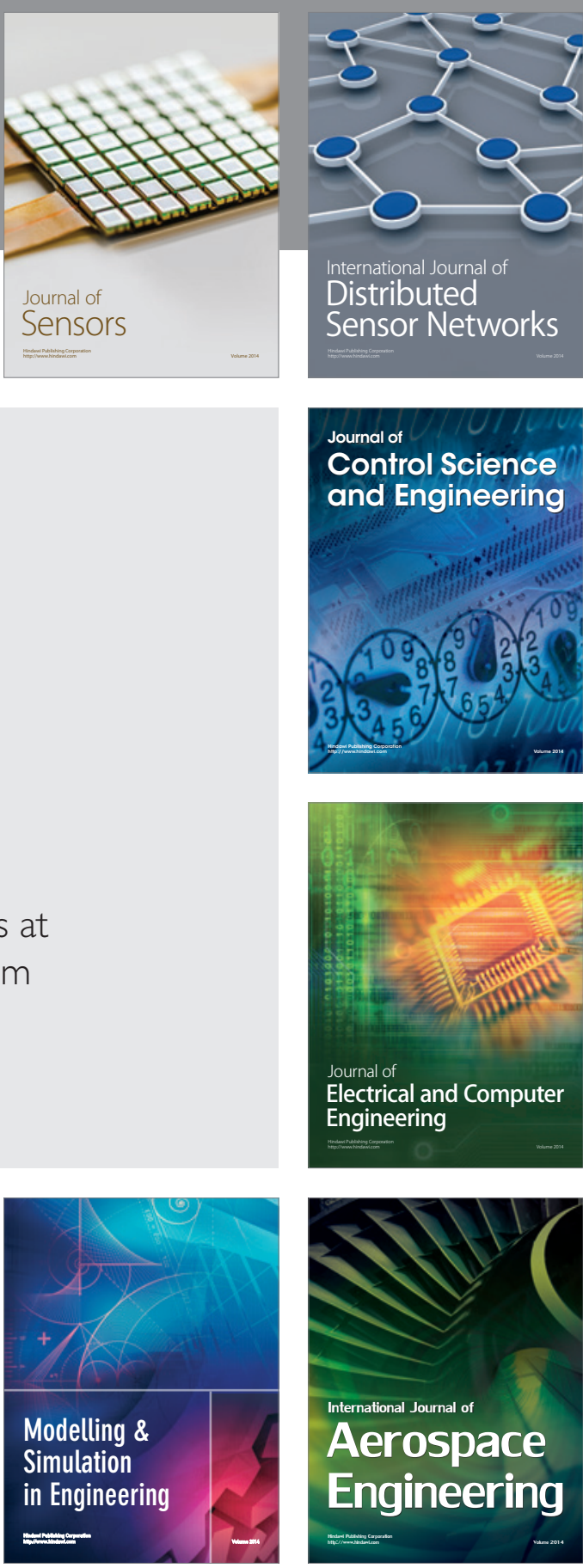

International Journal of

Distributed

Sensor Networks

$-$

Joumal of

Control Science

and Engineering
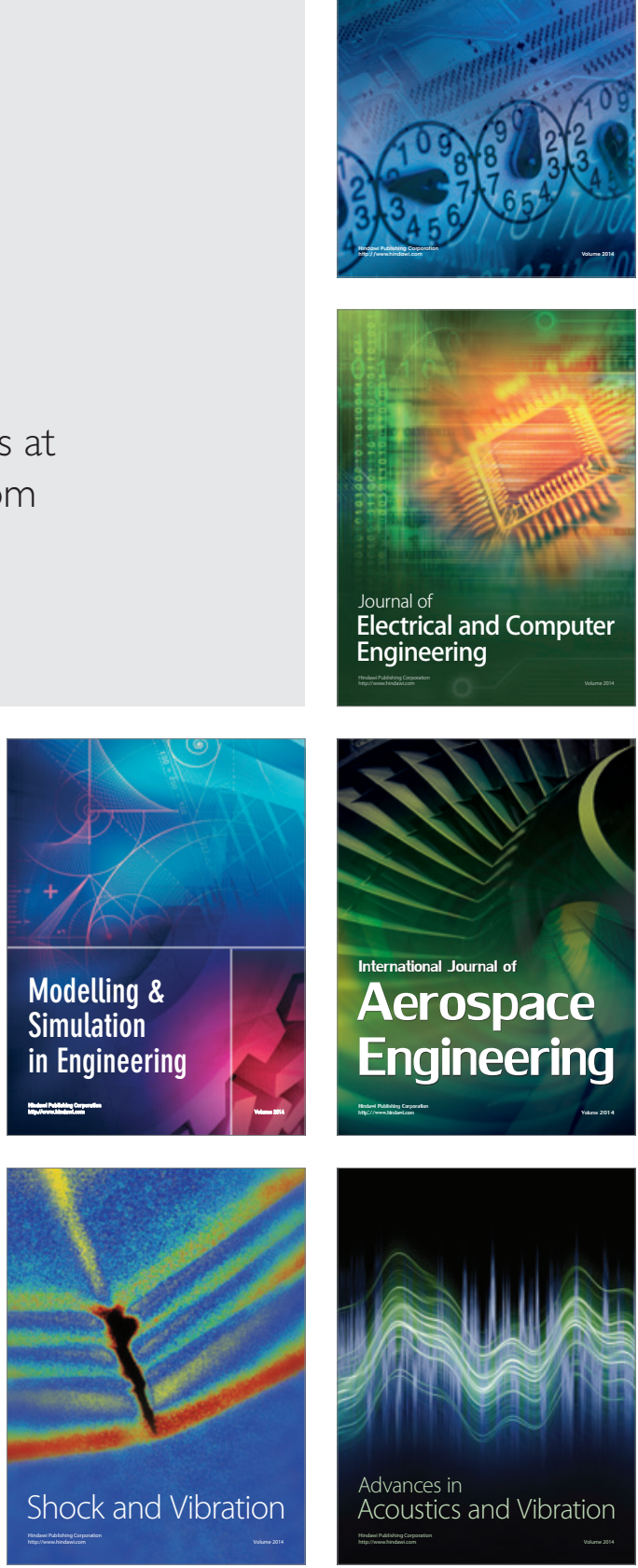